8. April 1909; dann ebendaselbst, im Valle Perla, an Kalkgestein in etwas feuchtem Buschwerk, ca. $10 \mathrm{~m} ; 14$. April 1909 . Auch über der Campora im Distrikte Gonaro findet sich die Pflanze, so in einer verwachsenen Gießbachschlucht (Ericetum) des Valle Gonaro (Gunar), Kalk, ca. $20 \mathrm{~m}$, c. per. und in einer Waldbachschlucht an der Grenze zwischen Gonaro und Mondanea, Kalk, ca. $50 \mathrm{~m}$; 22. April 1909.

Dieses stattliche, auffallende Lebermoos ist offensichtlich im westlichen Teile der Insel Arbe in feuchten, waldigen Einschnitten ziemlich verbreitet und tritt daselbst stellenweise reichlich auf. Anderwärts in Dalmatien scheint jedoch die Pflanze recht rar zu sein, sie findet sich weder in den Kollektionen Dr. Latzels aus der lebermoosreichen und von ihm genau durchsuchten Umgegend Ragusas, noch hat sie Baumgartner auf seinen über ganz Süddalmatien sich erstreckenden Exkursionen außer ein einziges Mal an einem im äußersten Süden gelegenen Standorte (Lissa) finden können.

32. Frullania dilatata (L.) Dum. - Insel Arbe: Dundo-Wald an Quercus Ilex, c. per. et $C^{\pi} ; 8$. April 1909. Valle Cifnata westlich von der Stadt Arbe, an Kalkblöcken, ca. $10 \mathrm{~m}$; 14. April 1909. -Insel Lussin: Monte Ossero südwestlich vom Gipfel, an Ostrya, Fraxinus Ornus. Acer monspessulanum, ca. 570 m, c. fr.; 26. April 1909. - Insel Veglia: Bei Malinska, an Paliurus-Rinde, ca. $50 \mathrm{~m}$; 2. April 1914. - Insel Cherso: Wald von Konec (Kunee) über Caisole, an alten Eichen, ca. $200 \mathrm{~m} ; 24$. März 1914.

33. Frullania Tamarisci (L.) Dum. - Insel Cherso: Wald von Konec (Kunec) über Caisole, an alten Eíchen, c. fr. und auf Erdboden, steril, ca. $200 \mathrm{~m} ; 24$. März 1914.

\title{
Untersuchungen über die ersten Entwicklungsstadien einiger Moose.
}

Von E. Lampa (Prag).

(Mit 30 Textfiguren.)

Wenn ich es unternehme, die nachstehenden Untersuchungen zu veröffentlichen, trotzdem diese in keiner Weise auf Vollständigkeit Anspruch erheben können, so mag als Entschuldigung und Begründung dafür gelten, daß diese Unvollständigkeit einerseits durch die Ungunst der Verbältnisse verschuldet ist, anderseits aber, wie ich glaube, die Klärung der zu besprechenden Frage nịcht sonderlich hindert. 
Die Untersuchungen erstrecken sich auf verschiedene. Moose, welche - Ricardia pinguis ausgenommen - im Gewächshause des botanischen Institutes der deutschen Universität in Prag, mit freundlicher Genehmigung des Herrn Prof. G. Beck von Mannagetta und Ler chenau, kultiviert wurden.

Die ausgesäten Sporen wurden mit aller Sorgfalt betreut, stets mit sterilem Wasser begossen ete.; trotzdem entwickelten sich die Kulturen höchst mangelhaft, zeigten Kümmerformen, und abgesehen von Sphagnum quinquefarium, gelang es mir bei keiner, sie bis zum ausgewachsenen Pflänzchen durchzubringen. Dazu mögen wohl die vielen nebeligen, finstern Tage der Winter $1909 / 10$ und 1910/11 beigetragen haben, hauptsächlich aber die im Prager Wasser überaus zahlreichen Algenkeime, die - vortrefflich an die gegebenen Verhältnisse angepaßt - sich in jeder Kultur überreichlich entwickelten und die keimenden Sporen der Moose geradezu erstickten, so dals jede Kultur nur wenige Pflänzehen ergab, die überhaupt zur Untersuchung geeignet waren. Wenn ich nun dieses mangelhafte Material doch verarbeite, so hat dies seinen Grund darin, daß ich an $\mathrm{hmm}$ neuerlich Beweise für ein den Bryophyten gemeinsames Bildungsgesetz ${ }^{1}$ ) oder, besser gesagt, eine immer wiederkehrende Gesetzmäßigkeit des Aufbaues in bestimmten Stadien erkennen konnte, die vielleicht der Mitteilung wert ist. Da es sich mir nur um die Betonung des Vorhandenseins dieser Stadien handelte, sebe ich auch von einer ausführlichen Darstellung der Entwicklung - die für die meisten Formen schon gegeben wurde - ab.

Der flächige Vorkeim von Sphagnum entwickelt sich verhältnismäßig rasch. An einem wenigzelligen Keimfaden entsteht eine Scheitelzelle in der Art, wie bei der Bildung eines Farnprothalliums; diese bildet mehrere Segmente, dann vergrößert sich das ganze Gebilde rasch mittels Randmeristem. (Abb. 3, Fig. 1-7.)

Das eigentliche Sphagnum-Pflänzchen entwickelt sich aus einer Randzelle der flächigen Vorkeime. Diese Randzelle wird zuerst besonders plasmareich; wölbt sich vor und teilt sich durch eine Wand in zwei Zellen. (Fig. 8.)

In einer dieser Zellen wird nun offenbar eine Scheitelzelle abgeschnitten und das weitere Wachstum des auf diese Weise angelegten Pflänzchens erfolgt genau wie in den ersten Stadien eines Lebermoospflänzchens (Fig. 9-12), dem es auch auffallend ähnlich sieht. Zuweilen

1) L a m p a E. in Sitzungsberichte der kaiserl. Akademie der Wissensch. in Wien. Bd. CXII, 1903.

$\mathrm{D}$ i $e \mathrm{~s} \in \mathrm{lb}$ e. Über die Beziehungen zwischen dem Lebermoosthallus und dem Farnproth. Österr. botan. Zeitschr., 1909. 
unterbleibt der flächige Vorkeim, dann ist die Äbnlichkeit um so stärker ausgesprochen. (Fig. 13 u. 14.)

Bei den Pfänzchen Abb. 3, Fig. 13, 14 besaßen die ältesten Blätter schon den typischen Charakter der Sphagnaceen. Auffallend ist das lang-
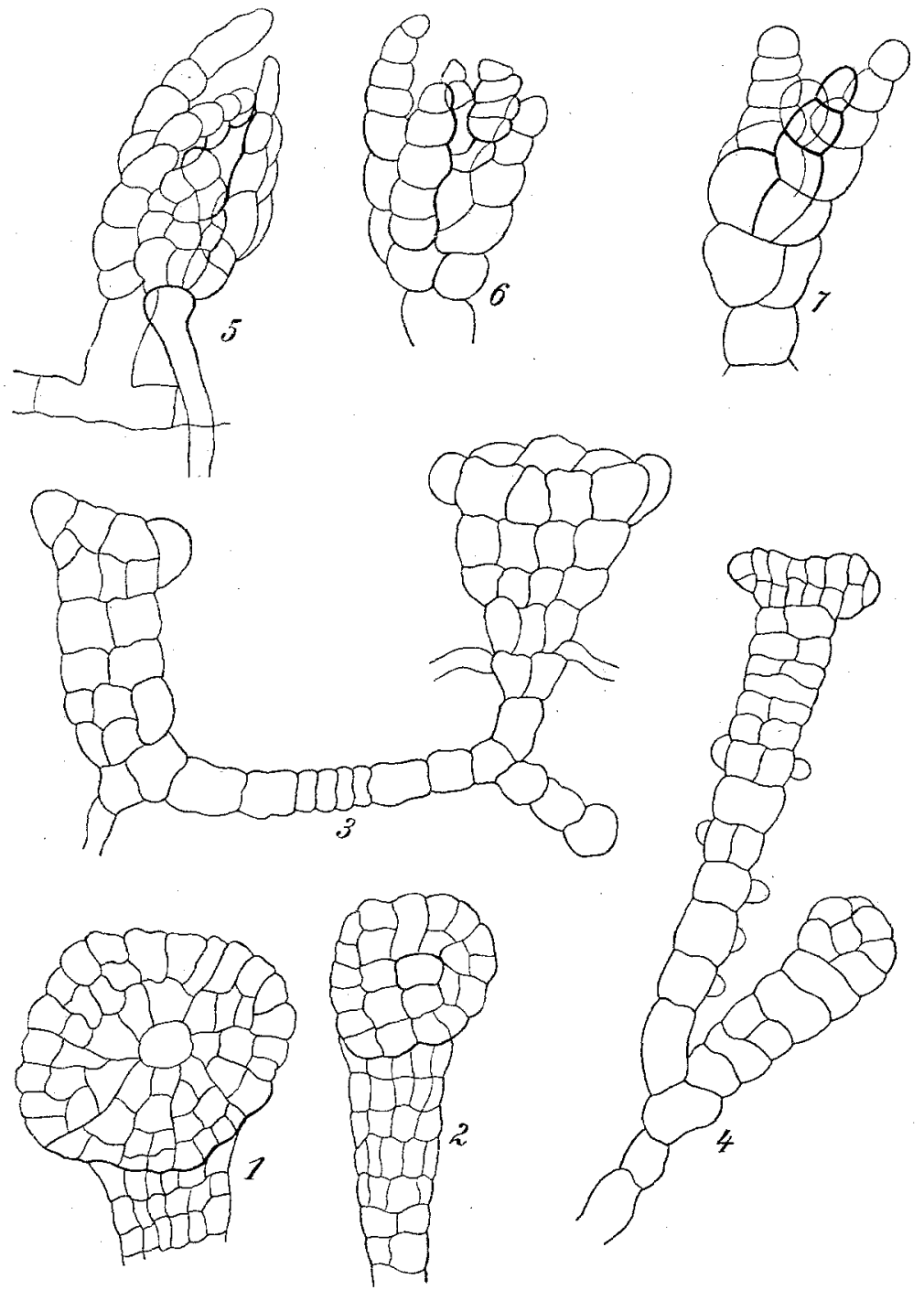

Abb. 1.

same Wachstum der Sphagnum-Pfänzchen; diese sind nach nunmehr zweijähriger Kultur kam länger als $1-1 \cdot 5 \mathrm{~cm}$. Vielleicht sind wegen dieses langsamen Wachstums die flächigen Vorkeime nötig, um die Lebensfähigkeit der ungemein zarten Knospen zu erhöhen. 
Die fadenförmigen Vorkeime von Haplomitrium Hookeri sind von denen eines Laubmooses überhaupt nicht zu unterscheiden. (Abb. 2, Fig. 1 u. 2.) Und nur der Vergleich von den Faden anhaftenden Sporen mit einigem aufbewahrten Material gab mir die Gewißheit, tatsächlich Haplomitrium vor mir zu haben. Der vielzellige Faden, der im Wesen von einem Laubmoosprotonema nicht verschieden ist, verzweigt sich hier viel reicher als bei Cephalozia, Nardia oder Lophocolea ${ }^{1}$ ), wo die Möglichkeit der Verzweigung gleichsam nur angedeutet ist. Er trägt schließlich häufig mehrere Pflänzchen. (Abb. 2, Fig. I, II, III, IV.)

Dieses Lebermoos, das im erwachsenen Zustande bekanntlich aufrecht steht und dreizeilige Beblätterung zeigt, hat in der ersten Anlage des Pflänżchens eine viel weniger ausgesprochene Beblätterung als viele Jungermanniaceen und selbst als dies etwa bei Chomiocarpon quadratus der Fall ist ${ }^{2}$ ).

Diphyscium foliosum entwickelt die bekannten keulenförmigen Gebilde (A bb. 1, Fig. 3 u. 4), deren auffallend regelmäßiger Aufbau von einer in der Daraufsicht (Fig. 1 u. 2) erkennbaren Scheitelzelle aus vor sich geht. Die eigentlichen Moospflänzchen stehen in keiner erkennbaren Beziehung zu diesen Keulen, doch hoffe ich durch Weiterkultur dieser Form in dieser Hinsicht noch Aufschluß zu erhalten. Sie besitzen eine deutlich erkennbare Scheitelzelle (Fig. 5) und gleich en jungen Knospen von Lophocolea heterophylla (Fig. 6) und Cephalozia bicuspidata (Fig. 7).

Schließlich möchte ich auf die große Ähnlichkeit zwischen der Anlage eines Sprosses von Ricardia pinguis mit dem eines Laubmooses hinweisen. Der Längsschnitt durch den Scheitel des fleischigen, ziemlich undifferenzierten Thallus einer allerdings nicht normalen Pflanze (Abb. 4, Fig. 3) zeigt eine unverkennbare Übereinstimmung mit dem Aufbau eines Laubmooses (Abb. 4, Fig. 4). Wir sehen demnach, daß die herangezogenen Formen, besonders in ihren Jugendstadien, gemeinsame Merkmale haben, die durchaus nicht notwendige Daseinsbedingungen sind, da sie ja auch indiriduell fehlen können und die zwanglos auf eine phylogenetische Zusammengehörigkeit schließen lassen. Zu den einzelnen Formen seien noch folgende Bemerkungen hinzugefügt:

\section{Haplomitrium Hookeri ${ }^{3}$ ). (Abb. 2.)}

Bei der Keimung der Spore wird das Exosporium gesprengt und die erste Zelle des Keimfadens tritt heraus. Wie ich schon erwähnte, wird dieser Zellfaden, das Protonema, sehr vielzellig und verzweigt. Fußnote 1.

1) L a mp a E. Untersuchungen an einigen Lebermoosen I. c. Siehe S. 196,

2) L a m p a E. Untersuchungen an einigen Lebermoosen. Sitzungsber. d. kais. Akad. d. Wissenseh. in Wien, Bd. CXI, 1902.

3) Vgl. L e itg e b H. Unters. üb. d. Lebermoose. II. 1875. 
Bei Abb. 2, Fig. 1 besaß der Faden, der ein Pflänzchen trug, mehr als 30 Zellen.

Das Exemplar Fig. 2 ist vielfach verzweigt und fast jeder Zweig trägt ein Keimpflänzchen II., III., IV.

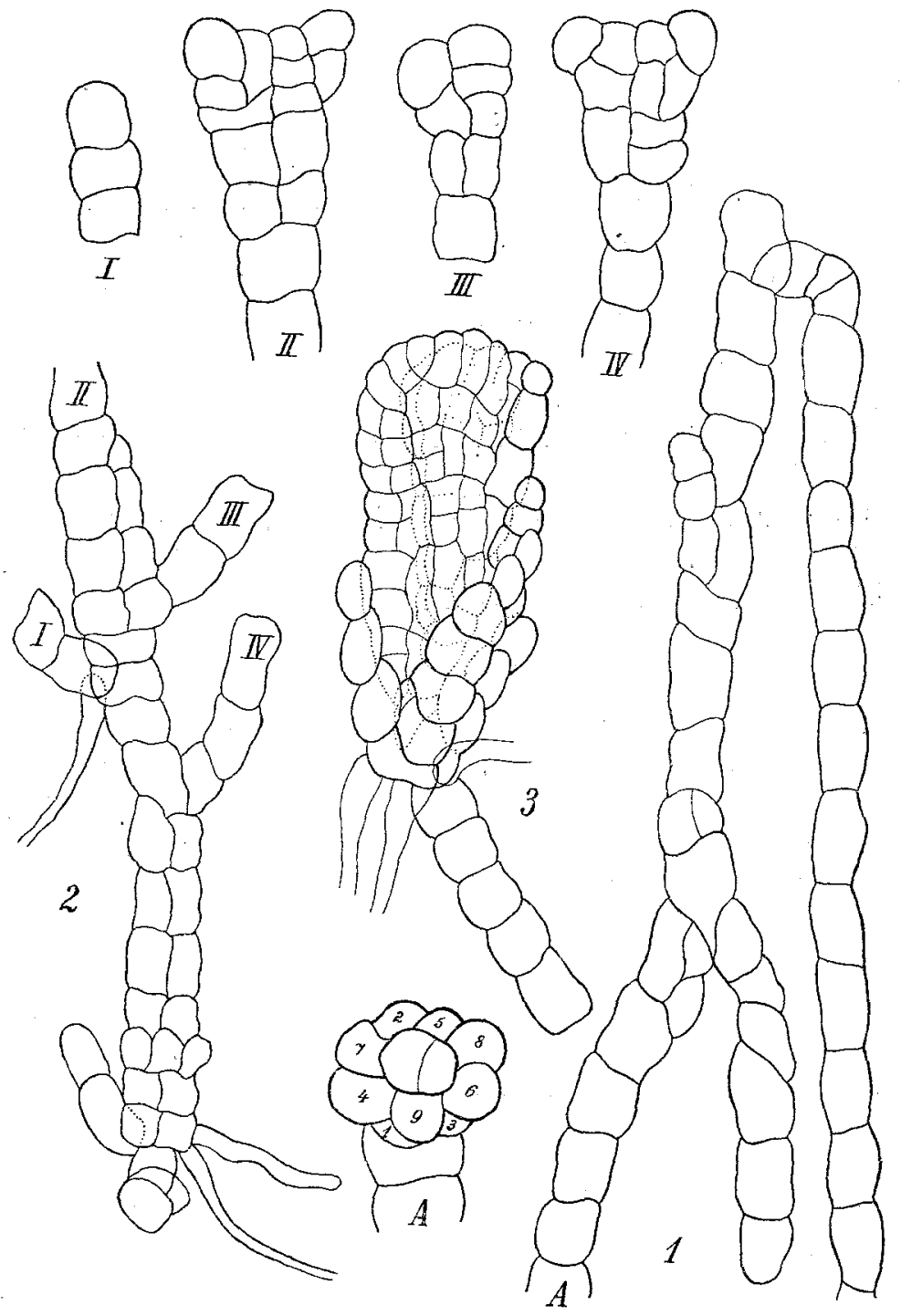

Abb. 2 .

Die jungen Pfänzchen, die sich als ziemlich undifferenzierte Gebilde entwickeln, zeigen immerhin erkennbare Segmentierung, jedoch durchaus keine Neigung zur Quadrantenteilung. Die von der Scheitel- 
zelle Fig. $A$ abgeschnittenen Segmente wachsen bald zu Blattrudimenten und endlich - in verbältnismäßig sehr frühem Stadium - zu Blättern aus. (Fig. 3.)

\section{Sphagnum quinquefarium ${ }^{1}$ ). (Abb. 3.)}

Bei Sphagnum quinquefarium ist der Keimfaden auf $1-3$ Zellen reduziert, wenn die Keimung auf feuchter Erde vor sich geht. Die Kultur der Sporen im Wasser mißlang; doch wäre die Anlage der Pflanzen an dem in diesem Falle fadenförmigen Vorkeim sicherlich nicht uninteressant gewesen. Der Vorkeim entwickelt sich nun, ähnlich wie ein Farnprothallium, zu einem flächigen, zuweilen herzförmigen Gebilde, dessen Wachstum erst von einer Scheitelzelle, ausgeht, dann mittels eines Randmeristems erfolgt (Fig. 1-7). Am unteren Rande des prothalliumartigen Gebildes entstehen nun, wie schon früher besprochen, die Sphagnum-Pfänzchen. (Fig. 8-12.)

Auf den papillenartigen Charakter der jüngsten Blätter möchte ich besonders hinweisen.

Offenbar als abnormale Fälle sind die Pflänzchen, durch Fig. 13 und 14 dargestellt, aufzufassen. Sie entstanden an kurzen Keimfäden mit Übergehung des flächigen Vorkeims.

\section{Ricardia pinguis.}

Das zur Untersuchung gelangte Material hatte ich nicht selbst kultiviert, sondern es war von Dr. E. Zederbauer in der Nähe von Reindlmühl bei Gmunden ausgegraben und mir überlassen worden. Die Pflänzchen hatten vollständig unterirdisch vegetiert, waren weiß gefärbt und anscheinend chlorophyllos, dabei ziemlich undifferenzierte Gebilde und nicht ohne weiteres zu identifizieren; Prof. V. Schiffner bestimmte sie als Ricardia pinguis. Die Zellen des dicken, fleischigen Vegetations. körpers waren dicht mit Pilzhyphen angefüllt. Pilzhyphen in Rhizoiden ${ }^{2}$ ) wurden schon vor ziemlich langer Zeit gefunden. A. J. M. Garjeanne untersuchte hauptsächlich foliose Jungermanniaceen und fand, daß Lebermoosverpilzung etwas Zufälliges und Inkonstantes sei, jedenfalls etwas häufig Vorkommendes. Der Grad der Verpilzung ist nach diesem Autor bei derselben Art und sogar beim selben Standort verschieden. Bei manchen Arten werden nur die Rhizoiden und Nachbarzellen infiziert, bei anderen dringen die Pilze auch in die Zellen der Stämmchen ein oder sie durchziehen die ganze Pflanze.

1) Betreffend die Literatur vgl. Warnst or $\mathbf{f}$ in $\mathbf{E} \mathrm{ng} \mathbf{l}$ e $\mathbf{r}-\mathbf{P r a n t l}$. Natürl. Pflanzenfam. I. 3. p. 248, 1909.

2) A. J. M. G arje a n ne, Die Verpilz. d. Lebermoosrhiz. Flora, Neue Folge, II. Bd., 1911. 


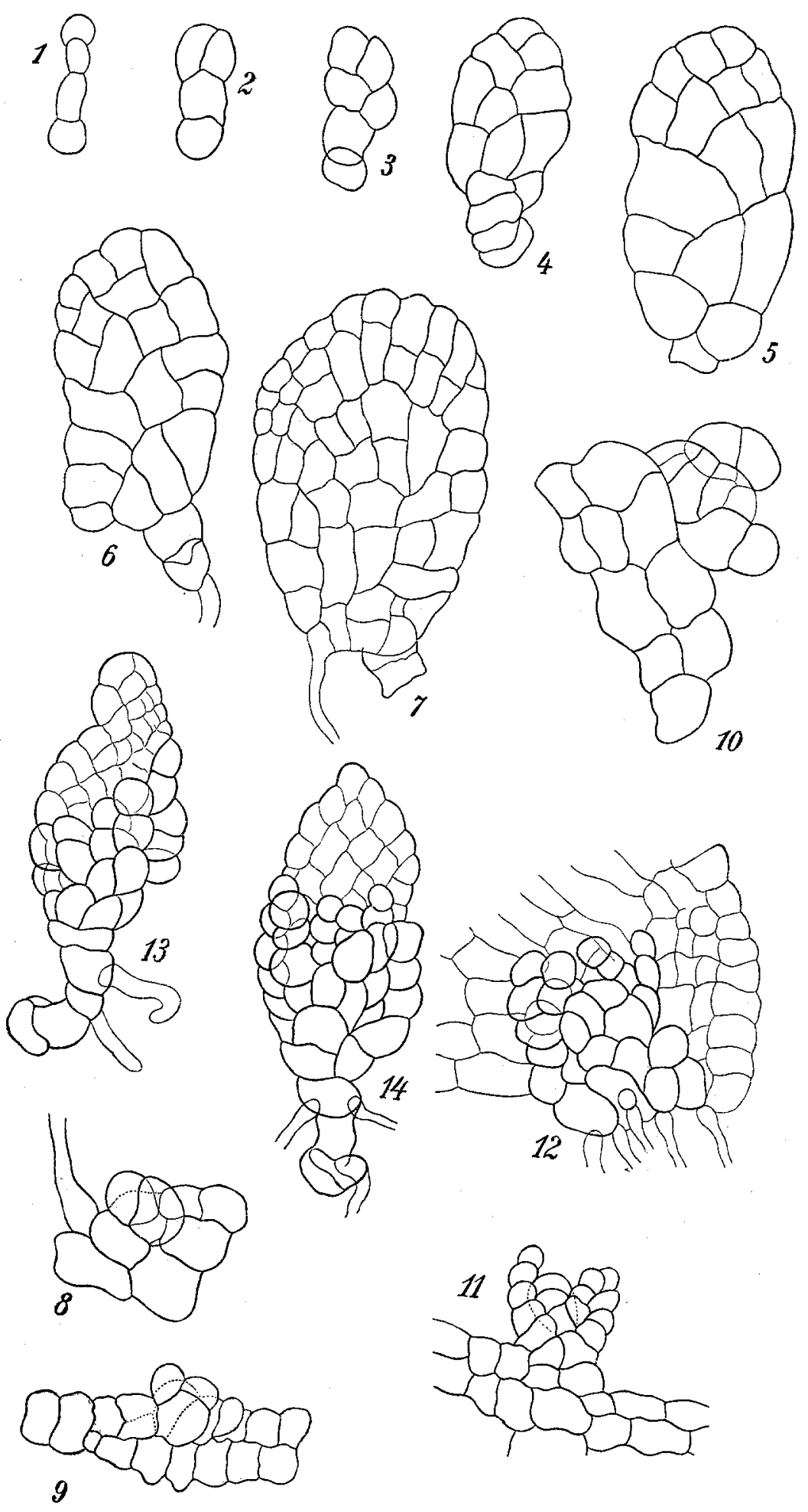

Abb. 3. 
Jaroslaw Peklo ${ }^{3}$, der in seiner Arbeit „Einiges über die Mycorrhiza bei den Muscineen" ziemlich viele Formen von Laub- und Lebermoosen untersucht und auch die Ergebnisse aus der einschlägigen Literatur recht eingehend berücksichtigt, kommt zu dem Schlusse, daß diese Symbiose für diese Pflanzengruppen nichts anderes sei als "ein harmloses - normale ''üchtigkeit beider Symbionten vorausgesetzt - der Wirtspflanze kaum nachträgliches Zusammenleben zweier Organismen, welches für den Pilz sicher vorteilhaft ist, der höheren Pflanze hingegen, wenn üborhaupt welchen, so nur geringen Nutzen bringt."

Allerdings handelte es sich bei den von Peklo untersuchten Formen immer um normale grüne Pflanzen. In dem von mir beobachteten Falle lebten alle gefundenen Individuen im Vereine mit dem Pilz, die ganze Pflanze war von den Hyphen durchzogen und in allen ihren Teilen von weißlicher Farbe und augenscheinlich chlorophyllos, so daß die Bedeutung des Pilzes für diese Form von Ricardia pinguis laum in Frage gestellt werden kann, da doch Ricardia pinguis sonst als normal assimilierende Pflanze bekannt ist. Peklo hebt sogar hervor, daß Ricardia pinguis (Aneura pinguis) nebst Blasia und Metzgeria von ihm stets mykorrhizenfrei gefunden wurde, während Grace L. Clapp ${ }^{2}$ ) in seiner Arbeit "The Life History of Aneura pinguis" auf das gelegentliche Auftreten von Pilzhyphen hinweist; auch bei ihm handelt es sich um normal aussehende, grüne Pflanzen.

Es scheint, daß der ron mir untersuchte Organismus durch seine Lebensweise in der Erde bei Lichtabschlus unbedingt auf die Zuführung von organischer Substanz durch den Pilz angewiesen ist. Offenbar ermöglicht die sonst ohne Notwendigkeit bestehende Symbiose in diesem bestimmten Falle dem Lebermoos jene Form des Daseins, in der es unter den gegebenen Verhältnissen überhaupt noch existieren konnte. Wie diese Verbältnisse sich herausgebildet haben, kann wohl kaum rekonstruiert werden. Die Einwanderung des Pilzes in die keimende Spore beginnt, sobald das Exosporium durch das keimende Protoplasma gesprengt wurde. Auch Clapp konstatierte bei ganz jungen Keimlingen in manchen Zellen Pilzknäuel.

Aus äußeren Gründen konnte das mir zur Verfügung stehende Material nicht im lebenden Zustande aufgearbeitet werden. Es ging leider zugrunde, und so konnten weder die merkwürdigen Pfänzchen weiter kultiviert noeh die dieselben erfüllenden Pilzhyphen isoliert werden. Am Spiritusmaterial konnte ich beobachten, daß in alle keimenden Sporen Pilzhyphen eindrangen und dac die Zellen der Päanzchen in allen Stadien mit Pilzhyphen versehen waren, die lose oder in Knäueln den Zellinhalt

1) Bulletin internat. de l'Academie des Sciences de Bohême, 1903.

2) The Botanical Gazette, Sept. 1912. 
erfüllen. Das Protoplasma ist meist zusammengeballt. Peklo hatte bei Fegatella conica die Auflösung der Stärke der Wirtspflanze durch ein vom Pilze ausgeschiedenes Enzym beobachtet. Ähnliches zu sehen war
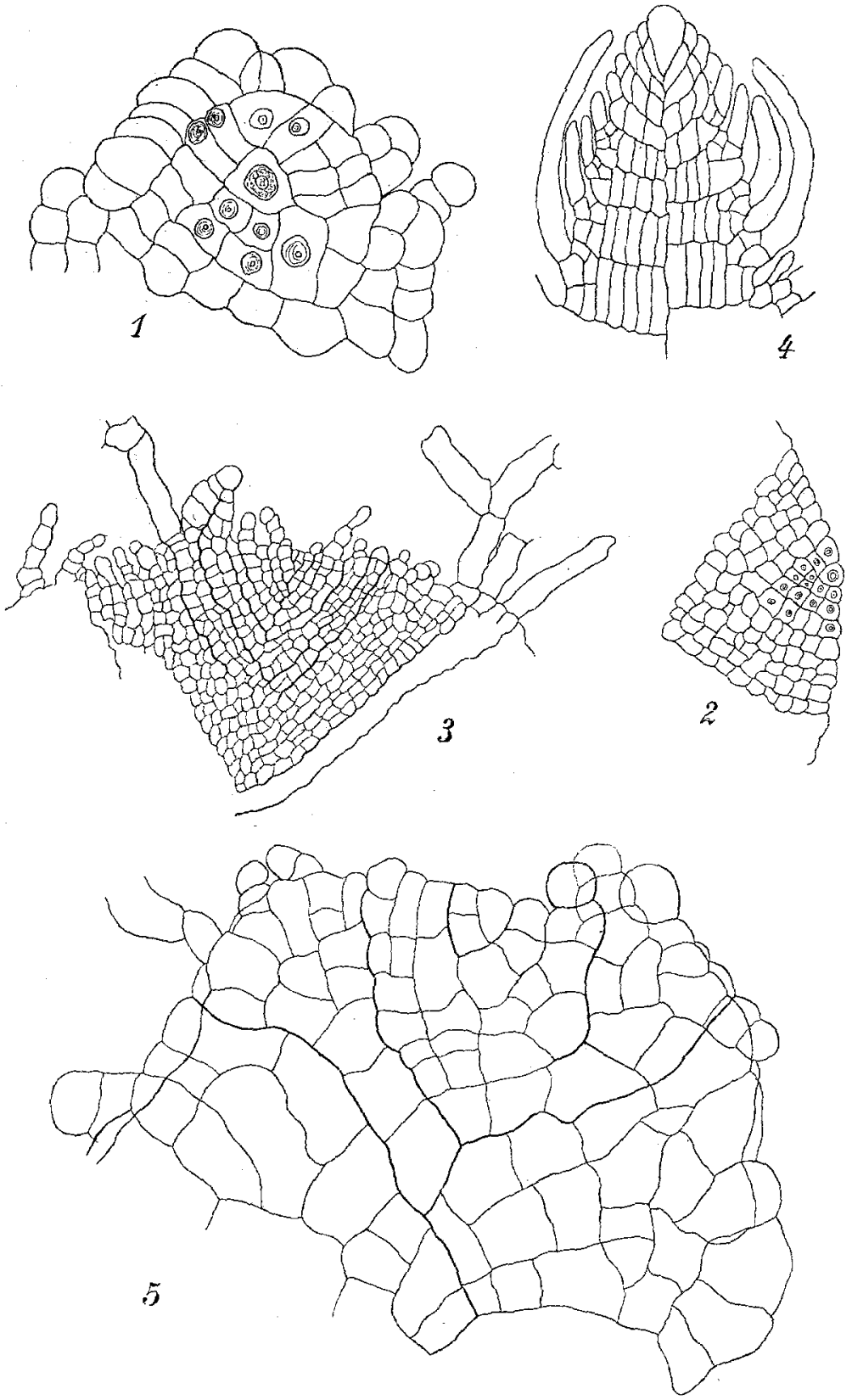

Abb. 4 . 
mir beim Spirituspräparat natürlich unmöglich, doch gestattet der $\mathrm{Zu}$ stand, in dem das Protoplasma sich in vielen Zellen befand, die Annahme, daß auch bier ein für den Pilz günstiges Ernährungsverhältnis bestehen muß.

Ich verweise schließlich auf die Scheitelzellenregion (Abb. 4, Fig. 1), die typisches Wachstum eines Lebermoospflänzchens andeutet, und auf den Längsschnitt durch ein junges (Abb. 4, Fig. 2 u. 5) und durch ein älteres Pfänzchen (Fig. 3); beide zeigen in ihrem oberen Teil und in der Scheitelregion dieselbe Gesetzmäßigkeit des Aufbaues ${ }^{1}$ ) wie ein Laubmoos (Abb. 4, Fig. 4) ${ }^{2}$ ), trotz anscheinender morphologischer Unterschiede. Clapp stellt für Aneura pinguis das häufige Vorkommen von zwei Scheitelzellen fest. Ich konnte dies nicht beobachten.

Ich glaube nun, daß, wie ich dies schon für andere Formen hervorgehoben habe, die besprochenen Pflanzen in ihren Jugendstadien manche Übereinstimmung zeigen, die keineswegs auf gleiche äußere Verhältnisse zurückzuführen ist, sondern darauf hinweist, daß, der später eintretenden morphologischen und biologischen Differenziernng ungeachtet, wir doch berechtigt sind, nach einem gemeinsamen Ausgangspunkt für die Organismengruppe der Laubmoose, der Lebermoose und der Farne zu suchen.

\section{Beiträge zur Kenntnis der Flora Kretas.}

Aufzählung der anläßlich der fünften Wiener Universitätsreise im April 1914 auf Kreta gesammelten Blüten. und Farnpflanzen.

Von Dr. Friedrich Vierhapper (Wien).

(Fortsetzung. ${ }^{3}$ )

(Mit Textfiguren.)

\section{Labiatae.}

336. Ajuga iva (L.) Schreb.

f. cleistogama Heldr. - S: Tybaki-Klima (Hö. We). - N : Candia (E); Knossos (We).

$\mathrm{Ob}$ es sich um die kleistogame Form der rosenrot oder gelb blühenden Rasse ( $A$. iva s. s. oder pseudoiva Rob. et Cast.) handelt, lälit sich wohl kaum entscheiden.

1) We t t s te in, Handbuch der syst. Botanik, 2. Aufl., S. 258.

2) Engler u. Prantl, Die natürl. Pflanzenfam. I. Teil, Abt. 3, 1. Hälfte, S. 172, Abb. 92.

3) Vgl. Österr. botan. Zeitschr. Bd. 64, 1914, S. 465-482, Bd. 65, 1915, S. 21 bis S. 28, S. $50-75$, S. $119-140$. 